\title{
A rare Von Hippel-Lindau disease that mimics acute myelitis: case report and review of the literature
}

\author{
Hong Jiang $\cdot$ Yu-ting Shi $\cdot$ Jun-ling Wang $\cdot$ \\ Bei-sha Tang $\cdot$ Jun-yu Wang $\cdot$ Ze-feng Peng • \\ De-sheng Xiao
}

Received: 3 November 2009/ Accepted: 1 September 2010/Published online: 7 October 2010

(C) The Author(s) 2010. This article is published with open access at Springerlink.com

\begin{abstract}
Von Hippel-Lindau disease (VHL) comprises a series of complicated clinical manifestations. We hereby report one unique case of VHL with a natural history that mimics acute myelitis. MRI and biopsy in this patient showed multiple solid hemangioblastomas of the central nervous system and kidney. This study further confirmed that VHL is of highly clinical, imaging, and pathological heterogeneity. Diagnosis for VHL should be based on combination of clinical, radiological, pathological, and genetic data.
\end{abstract}

Keywords Von Hippel-Lindau disease .

Hemangioblastoma - Clinical manifestation .

Magnetic resonance image $\cdot$ Histopathology

H. Jiang and Y. Shi contributed equally to this work.

H. Jiang $(\varangle) \cdot$ Y. Shi $\cdot$ J. Wang $\cdot$ B. Tang

Department of Neurology, Xiangya Hospital,

Central South University, 87\# Xiangya Road,

Changsha 410008, Hunan, People's Republic of China

e-mail: jianghong73868@yahoo.com.cn

H. Jiang - B. Tang

Neurodegenerative Disorders Research Center,

Central South University, Changsha 410008,

Hunan, People's Republic of China

J. Wang $\cdot$ Z. Peng

Department of Neurosurgery, Xiangya Hospital,

Central South University, Changsha 410008,

Hunan, People's Republic of China

D. Xiao

Department of Pathology, Xiangya Hospital, Central South University, Changsha 410008,

Hunan, People's Republic of China

\section{Introduction}

Von Hippel-Lindau disease (VHL), a multisystem hereditary neoplastic syndrome, most commonly presents with a cerebellar or retinal hemangioblastoma [1]. The disease is complex in its manifestation and therefore difficult to diagnose. We here present one unique case of VHL with a natural history that was initially misevaluated as acute myelitis, which together with literatures reviewed will be helpful for a better understanding of VHL.

\section{Case report}

A 28-year-old male presented with a 2-day history of acute and progressive back-neck pain, and numbness and weakness of both lower extremities concurrent with feces and urine retention secondary to an influenza 1 week before onset. He responded markedly to intravenous administration of glucocorticoid for 1 week after being initially misevaluated as "acute myelitis" in a poor countryside clinic, who was misdiagnosed by the barefoot doctor only according to his natural history without detailed examination by CT or MRI. On our body examination, muscle strength of lower limbs was grade $5^{-}$, with increase of their muscle tone and accentuation of deep tendon reflex. Banbinski sign and meningeal stimulation response were positive. Superficial and deep hypoesthesia below T9 were found.

Routine examinations, including cerebrospinal fluid, showed no abnormality except that abdominal ultrasound demonstrated renal lesions. Magnetic resonance brain, spinal cord, and kidney images showed multiple lesions of different size, mostly with clear boundaries and marked contrast enhancement (Fig. 1a-d). Spinal canal explorations 
Fig. 1 a Sagittal T2-weighted spine MR image. Two small nodular enhancing lesions with clear boundary at T10 and T12 were indicated (arrows).

b Sagittal T1-weighted cranialcervical MR image. Three oval enhancing lesions with clear boundary at the posterior medullar, the pontine base, and the intramedullary spinal cord at $\mathrm{C} 2$ were indicated (arrows). c Axial T1-weighted brain MR image. Two nodular enhancing lesions with clear boundary at the posterior medullar and the right cerebellar hemisphere were indicated (arrows). d Axial T1-weighted kidney MR image. A nodular enhancing lesion with unclear boundary in the lateral cortex of right kidney, as well as a circular-like enhancing lesion in the upper right kidney were indicated (arrows).

e Histopathology of the biopsied tissues from VHL patient. Many small blood vessels with a few foam cells, fat-like cells, and fibroblasts were indicated $(\times 400)$.

f Immunohistochemistry assay of the biopsied tissues from VHL patient. GFAP, F8, and Lys-positive cells were indicated (arrow, $\times 400)$
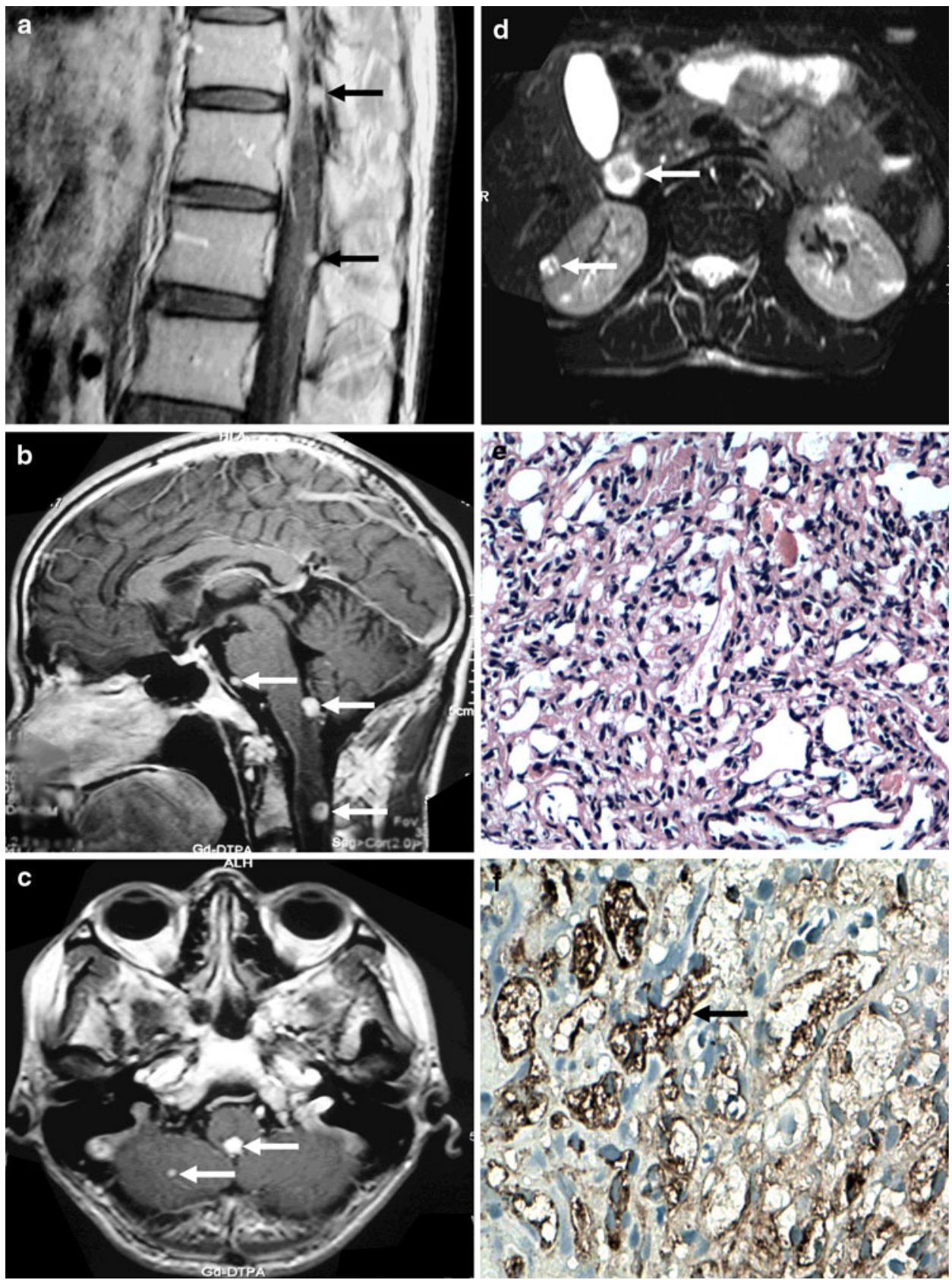

revealed two red nodular subdural lesions at T10 and T12 that accorded with Fig. 1a. Pathological analysis indicated many small blood vessels with a small amount of foam cells, fat-like cells and fibroblasts, which tested positive for GFAP, F8, and Lys, though negative for CD68, CK-pan, and EMA by immunohistochemistry assay (Fig. 1e, f). The mutation analysis was completed yet did not find the variation after polymerase chain reaction (PCR) and sequencing of three exons of $V H L$ gene. Systematic enquiry and examinations indicated his father as an asymptomatic affected individual with hemangioblastomas involved in brain and kidney. The patient improved further on his neurological manifestation 2 weeks after surgery on the two nodules. One year later, a follow-up study indicated that he was completely rehabilitated.

\section{Discussion}

The VHL is a rare $(1 / 36,000)$, autosomal dominant, neurocutaneous dysplastic condition [1, 2]. The following three criteria suggest a diagnosis of VHL: (1) one or more hemangioblastomas within the central nervous system (typically in the cerebellum), (2) presence of visceral 
lesions (e.g. renal, pancreatic tumors/cysts), and (3) familial incidence [1-4]. Common tumors found in VHL include retinal angiomas, CNS hemangioblastomas, renal cell carcinomas, and pheochromocytoma [1-6]. This patient was characterized by multiple tumors involved in both the central nervous system and visceral, as well as family history, which was in accordance with VHL criteria. Our case also exemplified a rare presentation of VHL as only a minority of hemangioblastomas will present in the spinal cord and even fewer in the cervical spine [3]. Furthermore, never has a case of VHL been reported that mimics onset form of acute myelitis.

Hemangioblastomas in CNS commonly occur in the posterior fossa and craniocervical junction [1, 2]. In this case, the brain lesions presented in posterior medullar adjacent to midmost foramina of the fourth ventricle, which had rarely been reported previously, and the spine lesions mainly occurred in the subdura of the cervical and thoracic spine in accordance with the previous report. It was reported that renal lesions are usually cysts or solid tumors, which are generally considered as malignant irrespective of the presentation of images. For this patient, renal tumors occurred in the early disease course, suggesting a high possibility of malignancy.

This patient had multiple lesions except retinal hemangioblastoma, whereas he indicated few of the corresponding symptoms, even without showing a chronic onset and course, which is rare in clinical practice, suggesting that VHL can be highly clinical heterogeneous. Thus, this case should be differentiated from acute inflammation or vascular disease.

Different from typical MRI features of VHL [3], this case showed a form of solid tumors with remarkable enhancement, yet without "flow-empty" phenomena, which should be distinguished from metastatic tumor, abscess, meningioma, vascular tumor, multiple small abscess, diffuse tuberculoma, or Schistosoma granuloma. Since VHL, in general, develops new lesions at about one lesion every 2 years, with known lesions having rapid growth, frequent follow-up MRI examinations seem necessary.

Of the histopathological features of VHL, one is the cytoplasmic vacuole-like structure formed via lipoid phagocytosis of tumor cells, another is plenty of reticular fibers distributed in tumors [4]. For this case, although the lesions had no cystic structure, the pathological findings complied with the typical changes of VHL.
The VHL gene on chromosome 3p25-26 was found in 1993, which acts as a tumor suppressor gene. Series of mutations in VHL have so far been identified. Although the genetic study did not find the mutation, it seemed that the disease might be heterogeneous. Currently we are applying new-generation sequencing technology to identify the novel VHL mutation in this patient for it might be due to a rare variant.

In sum, VHL is a rare multisystem neoplastic syndrome with complicated clinical manifestation. We reported one unique case of VHL with a natural history that mimics acute myelitis. Diagnosis for VHL should be based on combination of clinical, radiological, pathological, and genetic data. Early diagnosis, active radiological and surgical management, and frequent follow-up MRI examinations are critical to prognosis of VHL.

Acknowledgments We thank all the participants for their cooperation in this study. This work was supported by the National Natural Science Foundation of China (30971585, 30871354, 30710303061, and 30400262 to H.J.) and the Key Project of Hunan Natural Science Foundation (08JJ3048 to H.J.).

Open Access This article is distributed under the terms of the Creative Commons Attribution Noncommercial License which permits any noncommercial use, distribution, and reproduction in any medium, provided the original author(s) and source are credited.

\section{References}

1. Maher ER, Yates JR, Harries R et al (1990) Clinical features and natural history of von Hippel-Lindau disease. Q J Med 77:11511163

2. Conway JE, Chou D, Clatterbuck RE et al (2001) Hemangioblastomas of the central nervous system in von Hippel-Lindau syndrome and sporadic disease. Neurosurgery 48:55-63

3. Ho VB, Smirniotopoulos JG, Murphy FM et al (1992) Radiologicpathologic correlation: hemangioblastoma. Am J Neuroradiol 13:1343-1352

4. Weil RJ, Lonser RR, DeVroom HL et al (2003) Surgical management of brainstem hemangioblastomas in patients with von Hippel-Lindau disease. J Neurosurg 98:95-105

5. Altinoz MA, Santaguida C, Guiot MC et al (2005) Spinal hemangioblastoma containing metastatic renal cell carcinoma in von Hippel-Lindau disease. Case report and review of the literature. J Neurosurg Spine 3:495-500

6. Jarrell ST, Vortmeyer AO, Linehan WM et al (2006) Metastases to hemangioblastomas in von Hippel-Lindau disease. J Neurosurg 105:256-263 